\title{
A Delphi developed syllabus for the medical specialty of sport and exercise medicine
}

\author{
David Humphries, ${ }^{1}$ Rod Jaques, ${ }^{2,3}$ Hendrik Paulus Dijkstra, ${ }^{4}$ on behalf of the \\ International Syllabus in Sport and Exercise Medicine Group (ISSEMG)
}

${ }^{1}$ School of Medicine, University of Tasmania, Hobart, Tasmania, Australia

${ }^{2}$ Faculty of Sport and Exercise Medicine, Edinburgh, UK ${ }^{3}$ English Institute of Sport, London, UK

${ }^{4}$ Department of Sports Medicine, Aspetar Orthopedic and Sports Medicine Hospital, Doha, Qatar

Correspondence to Dr David Humphries, School of Medicine, University of Tasmania, Hobart, Tasmania 7000, Australia; sportsmedicine@outlook.com. $\mathrm{au}$

Accepted 30 November 2017 Published Online First 20 December 2017

Check for updates

To cite: Humphries $D$, Jaques R, Dijkstra HP, et al. Br I Sports Med 2018:52:490-492

\section{ABSTRACT}

Training in the medical specialty of sport and exercise medicine is now available in many, but not all countries. Lack of resources may be a barrier to the development of this important specialty field and the International Syllabus in Sport and Exercise Medicine Group was convened to reduce one potential barrier, the need to develop a syllabus. The group is composed of 17 sport and exercise medicine specialists residing in 12 countries (Australia, Canada, India, Ireland, Malaysia, the Netherlands, Qatar, South Africa, Sweden, Switzerland, the UK and USA). This paper presents the first phase of this project covering the domains and general learning areas of a specialist training syllabus in sport and exercise medicine.

\section{INTRODUCTION}

Sport and exercise medicine (SEM) has become a recognised field of medical specialisation in countries on six continents. The specialty of SEM includes the:

- promotion and implementation of regular physical activity in the prevention, treatment and rehabilitation of chronic diseases of lifestyle (termed 'noncommunicable diseases' by the WHO).

- prevention, diagnosis, treatment and rehabilitation of injuries that occur during physical activity.

- the prevention, diagnosis and management of medical conditions that occur during or after physical activity. ${ }^{1}$

In many countries SEM specialist training is not available. Physical inactivity poses significant health related risks throughout the world. Given the skill set that SEM physicians can bring in a variety of areas, including to any overarching governmental process to encourage and maintain physical activity, training of SEM physicians in these countries is highly desirable.

The development of a medical specialist training programme is a significant undertaking and requires considerable resources, both intellectual and physical. In 2016 a group of medical practitioners with experience in the development of training and assessment programmes in SEM were invited to join a Delphi group. The group was tasked with creating a 'basic syllabus in the specialty of SEM' with the intention that the syllabus be made available for use by any country wishing to develop a specialist training programme in SEM. It is hoped that by reducing the resource barrier more people, organisations and governments will have access to the expertise of specialist SEM physicians. The syllabus presented here is not intended for countries where specialty training is currently in place; excellent programmes already exist in these countries.

\section{BACKGROUND}

This project arose out of a series of discussions which occurred at SEM conferences in Australia, New Zealand and Europe in 2014 and 2015. The SEM specialist physicians involved in these discussions recognised that SEM is now practised at the specialist level in a substantial number of countries internationally, but that each country undertaking SEM specialist training had essentially 're-invented the wheel'. It was recognised that this was an inefficient use of scarce resources, and that the effort required to create a specialist training programme may form a barrier to the specialty being developed in more countries. The project is an attempt to provide an advanced starting point for those countries who do not have a SEM specialist training programme.

Internationally the existing postgraduate training path to specialty status for doctors in the field varies considerably. It ranges from undertaking an additional year or more of training postprimary specialty (eg, USA) through to stand-alone 4-year primary specialty training programmes (eg, Australia). Specialist SEM training is a lengthy process; in many countries training would not be complete before postgraduate year 7 .

The group is offering a syllabus rather than a curriculum as it was felt that the specialty will best evolve in individual countries on the back of pre-existing educational and medical infrastructure. The syllabus presented here is flexible enough to be used in a variety of specialist training scenarios. With an understanding of local resources, a national medical organisation could take this basic syllabus, and determine how they will train and assess in the specialty of SEM.

The authors recognise there are many upskilling short courses designed to improve a doctor's knowledge of various branches of SEM, however these do not produce SEM specialists.

No funding or organisational support has been sought for this project. The group has no affiliated organisations, although the individual group members have affiliations to various national SEM organisations.

\section{METHODS}

The Delphi group, which has come to be known as the International Syllabus in Sport and Exercise 
Medicine Group (ISSEMG) was formed by inviting approximately 20 SEM specialists from 12 countries where specialist SEM training is already established. The invitees were told of the nature of the project and asked to inform their national SEM organisation of the invitation, with the understanding that the national SEM organisations were not being asked to ratify the project, but could offer an alternate participant if the primary invitee could not participate for some reason, or if someone in the organisation had a greater interest in the project. Ultimately the group came to be composed of 17 SEM specialists residing in 12 countries (Australia, Canada, India, Ireland, Malaysia, the Netherlands, Qatar, South Africa, Sweden, Switzerland, the UK and USA).

The development process was a modified Delphi process, with questionnaires around topic inclusion being sent out at a rate of approximately one every 3 weeks, the comments of the group collated and circulated, with iterative questionnaires developed as needed. A cut-off point of $80 \%$ agreement was the general standard for topic inclusion. One member of the group (DH) created the questionnaires with multiple members offering initial lists of topic inclusions. Surveys were distributed via a link embedded in an email with a reminder email circulated approximately 2 weeks later. Response rates to the surveys varied from $60 \%$ to $100 \%$. Each questionnaire posed a series of questions around topic areas, with members agreeing or disagreeing on a topic's inclusion, with the option of commenting on the topic area and a final option of suggesting other topic areas for inclusion. The commentary and additional suggestions were then collated and circulated to the group with the option of further comment available. It was generally not possible for the collator to identify who had completed a survey or who had provided particular comments. Where appropriate, follow-up questions on the topic areas were posed in the next questionnaire.

The agreed syllabus is hierarchical, the top level contains the 'Domains', that is to say the overarching areas of learning. In the second level, the domains have been divided into 'General Learning Areas' (GLA) and each GLA will be divided into 'Specific Learning Areas' (SLA).

This paper presents the domains and GLAs; the SLAs are still in development and will be presented in a later publication.

It has been assumed that the doctors undertaking specialist training in SEM have prior high-level training in anatomy and exercise physiology. If this is not the case then these areas need to be included in the learning domains.

\section{RESULTS}

\section{Domains}

The following have been agreed as the key domains of SEM specialist training

1. Physical activity and human health

2. Medical issues related to exercise

3. Injuries related to sport and exercise

4. Nutrition

5. Pharmacology

6. Antidoping

7. Sports team care and sports event medical management

8. Physical activity in challenging environments

9. Specific groups undertaking sport and exercise

10. Intrinsic skills of an SEM physician

11. Extrinsic skills of an SEM physician

\section{General learning areas}

For each domain, the following have been agreed as the GLAs:
Physical activity and human health GLAs

1. The role of physical activity in the prevention and treatment of disease: population health perspectives

2. Applied exercise physiology: types of exercise, effects of exercise and maximising adaptations to exercise

3. Physical activity guidelines and recommendations

4. Barriers to physical activity: environmental, social, physical and psychological

5. Considerations before prescribing exercise

6. Exercise prescription in healthy individuals

7. Exercise prescription in individuals with disease

8. Exercise prescription in special circumstances

9. Communicating the physical activity message beyond the individual

Medical issues related to exercise GLAs

1. Neurological issues related to physical activity

2. Respiratory issues related to physical activity

3. Cardiovascular issues related to physical activity

4. Gastrointestinal issues related to physical activity

5. Renal and urogenital issues related to physical activity

6. Metabolic issues related to physical activity

7. Ear, nose and throat issues related to physical activity

8. Immunological and haematological issues related to physical activity

9. Dermatological issues related to physical activity

10. Psychological and mental health issues related to physical activity

Injuries related to sport and exercise GLAs

1. Principles of tissue injury and repair in the musculoskeletal system

2. Principles of injury prevention

3. General pathology of the musculoskeletal system

4. Head and neck injuries

5. Upper limb injuries

6. Trunk, abdominal and thoracic spine injuries

7. Lumbar spine and pelvic injuries

8. Lower limb injuries

9. Interpretation of radiological and other investigations

10. Principles of injury rehabilitation

11. Return to sport decision making

Nutrition GLAs

1. Sports nutrition for health and performance

2. Hydration

3. Carbohydrates

4. Fats

5. Protein

6. Micronutrients and vitamins

7. Energy requirements and relative energy deficiency

8. Nutritional supplements

Pharmacology GLAs

1. Medication abuse in elite athletes

2. The influence of medications used in the treatment of disease on exercise capacity

3. Medication and exercise interactions which may cause or worsen disease

Antidoping GLAs

1. The World Anti-Doping Authority (WADA) list

2. The WADA therapeutic use exemption process

3. Other prohibited medications in specific sports 
4. The consequences of doping: health risks, sanctions and responsibilities

Sports team care and sports event medical management GLAs

1. Roles of the SEM physician in the team environment

2. The Olympic movement medical code on the ethical treatment of athletes

3. Medical screening of athletes and event participants

4. Preseason/pre event medical organisation

5. Equipment, medical supplies and facilities for team and event care

6. Match/event day medical issues

7. Emergency sports medicine: on-field assessment and management of sports injuries and medical conditions

8. Postseason and postevent review of medical care

9. Team travel

10. Common general practice problems encountered when travelling with teams

Specific environments and sport GLAs

1. SEM as it relates to physical activity at altitude

2. SEM as it relates to physical activity in cold environments

3. SEM as it relates to physical activity in hot environments

\section{Specific groups in sport GLAs}

1. Sports medicine as it relates to paediatric athletes

2. Sports medicine as it relates to female athletes

3. Sports medicine as it relates to ageing athletes

4. Sports medicine as it relates to athletes with a disability

5. Sports medicine as it relates to extreme and adventure sport athletes

\section{Intrinsic skills of an SEM physician GLAs}

Intrinsic skills are core skills which all physicians should learn during basic training, but which also have particular applications within SEM practice.

1. Communication

2. Collaboration

3. Leadership and management

4. Health advocacy

5. Research, teaching and learning

6. Professionalism

7. Ethics

8. Cultural, religious and LGBTQ awareness and safety

Extrinsic skills of an SEM physician GLAs:

The ISSEMG have defined extrinsic skills primarily as the 'doing' skills, that is to say skills that require hands-on ability as opposed to the primarily cognition skills defined in the intrinsic skills.

Please note that in this domain the ISSEMG has chosen to define some skills as core, that is to say required of all specialists in SEM, and some skills as advanced, that is to say skills which could reasonably be expected to be acquired postspecialty training, but which could be acquired during specialty training.

Core skills

1. Perform a comprehensive examination of the musculoskeletal and neurological systems and interpret the findings at an advanced level.
2. Perform a sport-specific medical and musculoskeletal screening examination.

3. Perform advanced life support in non-hospital environments.

4. Provide effective immediate medical care for on-field injuries and medical events.

5. Perform concussion screening examinations, baseline and postinjury, and interpret the results.

6. Interpret radiological and other investigations relating to SEM at an advanced level.

7. Interpret ECG findings in an athlete with reference to current guidelines.

8. Inject a variety of joints and soft tissues without radiological guidance.

9. Prescribe advanced protective braces.

Advanced skills

1. Tape joints, tendons and muscle for injury prevention and treatment.

2. Interpret simple video analysis of a variety of sporting skills including running gait.

3. Perform a targeted ultrasound examination of a peripheral musculoskeletal problem.

4. Inject a variety of joints and soft tissues with radiological guidance.

5. Perform and interpret the findings of a resting and exercise lung function test.

\section{CONCLUSION}

ISSEMG has developed a baseline syllabus SEM medical specialist training programme and offers the first two layers of the syllabus in this document. The members of ISSEMG hope that this project is of value to those national medical organisations seeking to create a specialist training programme in SEM. ISSEMG intends to provide the SLA component of the syllabus within the next 3 years.

Acknowledgements The authors thank the ISSEMG team members for their contribution.

Collaborators Assoc Prof Irfan M Asif (USA), Prof Mark Batt (UK), Prof Mats Borjesson (Sweden), Dr H Paul Dijkstra (Qatar), Prof Bert Fields (USA), Dr Boris Gojanovic (Switzerland), Dr David Humphries (Australia), Prof Christa Janse Van Rensburg (Sth Africa), Dr Rod Jaques (UK), Prof Nahar Azmi Mahomed (Malaysia), Assoc Prof Margot Mountjoy (Canada), Dr Tvisha Parikh (India), Dr Diana Robinson (Australia), Assoc Prof Robert Sallis (USA), Prof Martin Schwellnus (Sth Africa), Dr Padraig Sheeran (Ireland), Dr Els Stolk (Netherlands).

Contributors All listed authors have contributed substantially to the concept design, writing and review of this paper. The collaborators listed were involved in the Delphi process which produced the data compiled in this paper.

Competing interests None declared.

Provenance and peer review Not commissioned; externally peer reviewed.

(c) Article author(s) (or their employer(s) unless otherwise stated in the text of the article) 2018. All rights reserved. No commercial use is permitted unless otherwise expressly granted.

\section{REFERENCE}

1 Schwellnus M, IOC Medical Commission, International Federation of Sports Medicine. The Olympic textbook of medicine in sport. Oxford, UK; Hoboken, NJ: Wiley-Blackwell, 2008. 